\title{
A case of maternal uniparental disomy of chromosome 20 detected by noninvasive prenatal test of 1,000 high-risk pregnancies
}

\author{
Dong Hyun Cha', Junnam Lee ${ }^{2}$, Young-Joo Jeon², Yong Wook Jung', Ja-Hyun Jang ${ }^{2,3}$, Taeheon Lee ${ }^{2}$, and Eun Hae Cho ${ }^{2, *}$ \\ ${ }^{1}$ Department of Obstetrics and Gynecology, CHA Gangnam Medical Center, CHA University, Seoul, Korea \\ ${ }^{2}$ Green Cross Genome, Yongin, Korea \\ ${ }^{3}$ Green Cross Laboratories, Yongin, Korea
}

Chromosomal loss in trisomy (trisomy rescue) to generate a disomic fetus can cause confined placental mosaicism and/or feto/ placental mosaicism. After trisomy rescue event, there is a risk of fetal uniparental disomy (UPD). Noninvasive prenatal test (NIPT) reflects the genomic constitution of the placenta, not of the fetus itself. Feto-placental discrepancy can therefore cause false-positive (trisomy) NIPT results. These discordant NIPT results can serve as important clues to find UPD associated with confined placental mosaicism. We report a case with maternal UPD of chromosome 20, detected by NIPT of 1,000 high-risk pregnancies, carried out for detecting chromosomal abnormalities in Koreans.

Key words: Uniparental disomy 20, Noninvasive prenatal test.

\section{Introduction}

Chromosomal loss in trisomy (trisomy rescue) to generate a disomic fetus can cause confined placental mosaicism and/or feto/placental mosaicism. After trisomy rescue event, there is a risk of fetal uniparental disomy (UPD). The phenotypic consequences of UPD for several chromosomes are still unknown or poorly understood. Therefore, work up for UPD is rarely performed, as a result of cytogenetic findings such as confined placental mosaicism, and apparently balanced translocation or unexpected homozygosity for a recessive allele.

Noninvasive prenatal test (NIPT) reflects the genomic constitution of the placenta, not of the fetus itself. Feto-placental discrepancy can cause false-positive (trisomy) NIPT results.
Therefore, these discordant NIPT results can provide important leads to find UPD associated with confined placental mosaicism. Brady et al. [1] recommended the follow up discordant NIPT and invasive testing with UPD work up, particularly in cases where chromosomes $6,7,11,14,15$, and 20 are involved because of the presence of known imprinting disorders related to these chromosomes. The maternal UPD 20 is substantially rare and the affected fetuses have a common feature of prenatal or postnatal growth delay [2-4]. Brady et al. [1] reported a total of 11 other chromosomal trisomies, involving all chromosomes except chromosomes 21, 18, and 13, detected using 4,000 NIPTs. Among them, six cases were followed up and four cases (two cases of trisomy 7 and one case each of trisomy 8 and trisomy 22) were revealed to have normal fetal karyotype in the amniotic fluid

Received: 12 April 2017, Revised: 28 May 2017, Accepted: 29 May 2017, Published: 30 June 2017

${ }^{*}$ Corresponding author: Eun Hae Cho, M.D., Ph.D.

Green Cross Genome, 107 Ihyeon-ro 30beon-gil, Giheung-gu, Yongin 16924, Korea.

Tel: +82-31-260-9216, Fax: +82-31-260-0620, E-mail: drchoeh@gmail.com

Conflict of interest: The authors declare that they do not have any conflicts of interest.

(c) This is an open-access article distributed under the terms of the Creative Commons Attribution Non-Commercial License (http://creativecommons.org/licenses/by-nc/4.0/) which permits unrestricted non-commercial use, distribution, and reproduction in any medium, provided the original work is properly cited.

c) Copyright 2017 by the Korean Society of Medical Genetics and Genomics 
and the other cases showed fetal mosaic trisomy 15, with UPD 15 and trisomy 16 mosaicism, respectively [1].

\section{Case}

In this study, we performed whole genome sequencingbased NIPT (G-NIPT ${ }^{\mathrm{TM}}$; Green Cross Genome, Yongin, Korea) of 1,000 high-risk Korean pregnancies, and detected five cases of chromosomal trisomies other than in chromosomes 21,18 , and 13: one case each of chromosome $7,8,16$, and two cases of chromosome 20. All three trisomy cases involving chromosomes 7 and 20 were investigated invasively for the presence of UPD. The case of trisomy 20 was revealed to have low level of mosaic trisomy $20(47, \mathrm{XX},+20[2] / 46, X X[40])$ at amniocentesis, but the other cases showed normal karyotypes. To rule out UPD, we performed chromosomal microarray analysis of the amniotic fluid and maternal genomic DNA in the three NIPT false-positive cases of trisomy 7 and 20, using Affymetrix CytoScan 750K array (Life Technologies, Carlsbad, CA, USA) that had 550,000 nonpolymorphic markers and 200,436 single nucleotide polymorphisms (SNPs). We found a 30-Mb homozygous region spanning the centromere, via amniocentesis of the fetus with low-level mosaic trisomy 20 (Fig. 1). The pattern of presence of the homozygous region limited to the centromere is consistent with trisomy rescue after meiosis II error, with evidence of crossing over. This finding suggests the occurrence of non-disjunction during meiosis. Subsequently, we compared all the genotypes for SNPs along the involved chromosomes, between the mother and the fetus. The maternal genome was concluded to be the origin of UPD, because fetal SNP genotypes in the 30-Mb homozygous region were identical with the maternal alleles. The case of mosaic low-level trisomy 20 was thus diagnosed to have maternal UPD 20, while the others did not show any UPD patterns.

\section{Discussion}

Only a limited number of cases have been reported with UPD 20, and most cases were postnatal [5]. Paternal UPD 20 presents pseudohypoparathyroidism type lb (OMIM 603233), characterized by hypocalcemia, hyperphosphatemia, and abnormally high parathyroid hormone levels. Maternal UPD 20 is related to structural alterations resulting in partial or complete trisomy for chromosome 20. The first case of maternal UPD showed a mosaic karyotype with $46, X Y$ and $47, X Y$, + mar, consisting of the centromere and pericentromeric segments for chromosome 20 [5]. The second case was prenatally diagnosed as mosaic trisomy 20 and postnatally revealed as nonmosaic maternal UPD 20 [2]. The third case had trisomy 20 in 98\% of the amniotic fluid, $100 \%$ of the placenta and urine sediment, and 10\% of the peripheral blood [4]. The fourth case, without identified trisomic cells, had intrauterine growth restriction (IUGR) and postnatal growth delay [6]. According to a recent report, maternal UPD 20 has been suggested to be a new imprinting disorder with IUGR, short stature, and prominent feeding difficulties with failure to thrive [7]. To best of our knowledge, two cases of UPD were detected by following discordant NIPT and invasive testing. Of these, one was a case of UPD 21 [8], and the other was a case of UPD 15 with fetal mosaicism [1]. In this study, we showed the potential use of NIPT for the detection of UPD, especially when the trisomies of specific chromosomes with imprinting syndromes are detected.

\section{Acknowledgements}

This work was supported the Post-Genome Technology Development Program (10053626, Development of Decision Making System for Emergency Prediction) funded by the Ministry of

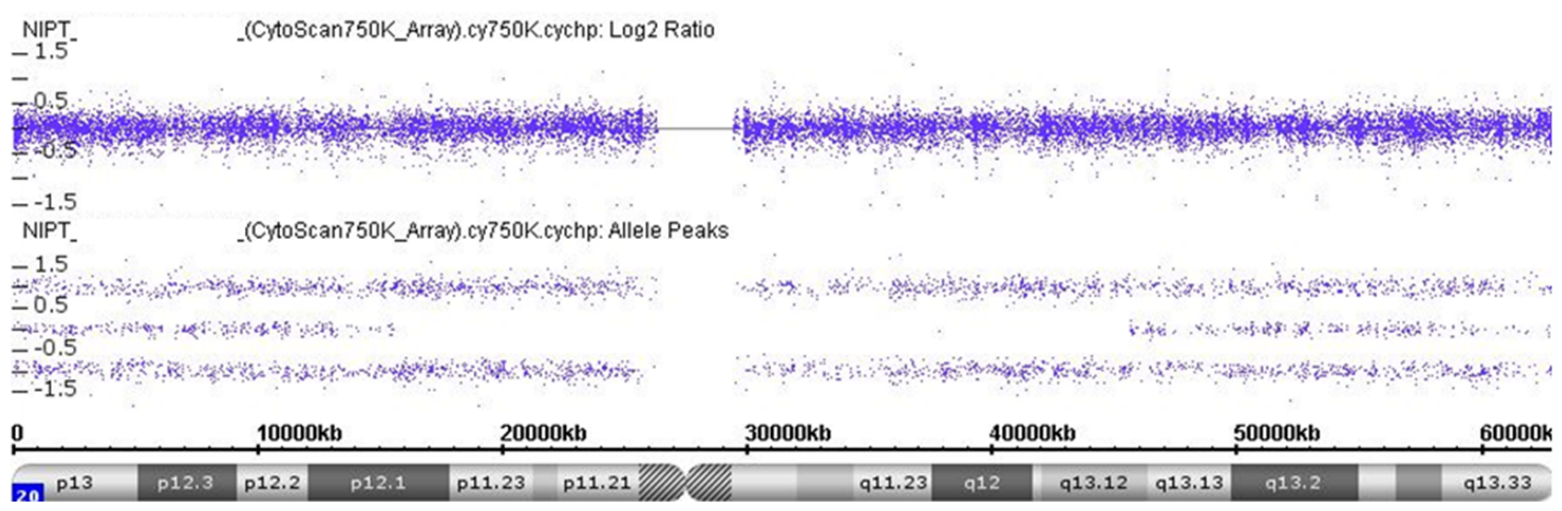

Fig. 1. Microarray analysis of amniotic fluid from a case of trisomy 20 detected by noninvasive prenatal test. The upper picture (Log2 ratio) shows normal two copy numbers of chromosome 20, and the lower line (allele peaks) shows centromeric 30-Mb loss of heterozygosity. 
Trade, Industry and Energy (MOTIE, Korea).

\section{References}

1. Brady P, Brison $N$, Van Den Bogaert $K$, de Ravel T, Peeters $H$, Van Esch $H_{\text {, }}$ et al. Clinical implementation of NIPT - technical and biological challenges. Clin Genet 2016;89:523-30.

2. Chudoba I, Franke Y, Senger G, Sauerbrei G, Demuth S, Beensen V, et al. Maternal UPD 20 in a hyperactive child with severe growth retardation. Eur J Hum Genet 1999;7:533-40.

3. Eggermann T, Mergenthaler S, Eggermann K, Albers A, Linnemann K,

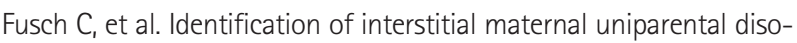
my (UPD) (14) and complete maternal UPD(20) in a cohort of growth retarded patients. J Med Genet 2001;38:86-9.

4. Salafsky IS, MacGregor SN, Claussen U, von Eggeling F. Maternal UPD 20 in an infant from a pregnancy with mosaic trisomy 20. Prenat
Diagn 2001;21:860-3.

5. Venditti CP, Hunt P, Donnenfeld A, Zackai E, Spinner NB. Mosaic paternal uniparental (iso)disomy for chromosome 20 associated with multiple anomalies. Am J Med Genet A 2004;124A:274-9.

6. Velissariou V, Antoniadi T, Gyftodimou J, Bakou K, Grigoriadou M, Christopoulou S, et al. Maternal uniparental isodisomy 20 in a foetus with trisomy 20 mosaicism: clinical, cytogenetic and molecular analysis. Eur J Hum Genet 2002;10:694-8.

7. Mulchandani S, Bhoj EJ, Luo M, Powell-Hamilton N, Jenny K, Gripp KW, et al. Maternal uniparental disomy of chromosome 20: a novel imprinting disorder of growth failure. Genet Med 2016;18:309-15.

8. Pan M, Li F, Li Y, Jiang FM, Li DZ, Lau TK, et al. Discordant results between fetal karyotyping and non-invasive prenatal testing by maternal plasma sequencing in a case of uniparental disomy 21 due to trisomic rescue. Prenat Diagn 2013;33:598-601. 